\title{
Topical Anti-Inflammatory Effects of Isorhamnetin Glycosides Isolated from Opuntia ficus-indica
}

\author{
Marilena Antunes-Ricardo, Janet A. Gutiérrez-Uribe, \\ Carlos Martínez-Vitela, and Sergio O. Serna-Saldívar \\ Centro de Biotecnología-FEMSA, Tecnológico de Monterrey, Avenida Eugenio Garza Sada 2501 Sur, 64849 Monterrey, NL, Mexico \\ Correspondence should be addressed to Janet A. Gutiérrez-Uribe; jagu@itesm.mx
}

Received 9 October 2014; Revised 15 December 2014; Accepted 16 December 2014

Academic Editor: Maxim E. Darvin

Copyright ( 2015 Marilena Antunes-Ricardo et al. This is an open access article distributed under the Creative Commons Attribution License, which permits unrestricted use, distribution, and reproduction in any medium, provided the original work is properly cited.

\begin{abstract}
Opuntia ficus-indica (OFI) has been widely used in Mexico as a food and for the treatment of different health disorders such as inflammation and skin aging. Its biological properties have been attributed to different phytochemicals such as the isorhamnetin glycosides which are the most abundant flavonoids. Moreover, these compounds are considered a chemotaxonomic characteristic of OFI species. The aim of this study was to evaluate the effect of OFI extract and its isorhamnetin glycosides on different inflammatory markers in vitro and in vivo. OFI extract was obtained by alkaline hydrolysis of OFI cladodes powder and pure compounds were obtained by preparative chromatography. Nitric oxide (NO), cyclooxygenase-2 (COX-2), tumor necrosis factor- (TNF-) $\alpha$, and interleukin- (IL-) 6 production were measured. NO production was tested in lipopolysaccharide-stimulated RAW 264.7 cells while in vivo studies were carried on croton oil-induced ear edema model. OFI extract and diglycoside isorhamnetin-glucosylrhamnoside (IGR) at $125 \mathrm{ng} / \mathrm{mL}$ suppressed the NO production in vitro $(73.5 \pm 4.8 \%$ and $68.7 \pm 5.0 \%$, resp.) without affecting cell viability. Likewise, IGR inhibited the ear edema (77.4 $\pm 5.7 \%)$ equating the indomethacin effects $(69.5 \pm 5.3 \%)$. Both IGR and OFI extract significantly inhibited the COX-2, TNF- $\alpha$, and IL- 6 production. IGR seems to be a suitable natural compound for development of new anti-inflammatory ingredient.
\end{abstract}

\section{Introduction}

The skin is the first line of defense against the external environment and xenobiotic agents. It interacts with both endogenous and environmental oxidant species which are involved in the pathogenesis of many inflammatory skin diseases [1-4]. Cutaneous injury causes keratinocyte activation, proliferation, and releasing of inflammatory mediators such as nitric oxide (NO), tumor necrosis factor alpha (TNF- $\alpha$ ), and interleukin- (IL-) 1 and IL-6 $[5,6]$.

Different inflammation models have been used to understand the anti-inflammatory mechanisms of synthetic drugs or compounds from natural sources [7]. Lee et al. [8] and Yoon et al. [9] observed that the extracts of Chrysanthemum indicum and Scutellaria baicalensis induced antiinflammatory responses in vivo and in vitro. These effects were evidenced by a reduction on the NO production, cytokines, chemokine, and growth factors, along with different histopathological parameters. Additionally, topical formulations which combine naturally occurring compounds and nutritional supplements have been developed and these have shown great potential to inhibit oxidative stress and skin inflammation [10].

The ear edema inflammation model has been widely used to evaluate the anti-inflammatory potential of natural compounds. The topical application of croton oil as an irritant agent increases vascular permeability and induces the synthesis of arachidonic acid metabolites and the expression of COX-2, IL- $1 \beta$, TNF- $\alpha$, and the adhesion molecule ICAM- 1 [11].

Different flavonoids-rich extracts and isolated flavonoids have been tested in vitro and in vivo to evaluate their anti-inflammatory effects. For example, a flavonoid-enriched fraction obtained from Cayaponia tayuya roots $(0.5 \mathrm{mg} / \mathrm{ear})$ showed an inhibition of $66 \%$ in acute TPA-induced edema in mouse ears. When the extract was tested at $22.30 \mu \mathrm{g} / \mathrm{ml}$ 
on RAW 264.7 macrophages, it inhibited the expressions of iNOS and COX-2 by $97 \%$ and $65 \%$, respectively [15]. Likewise, flavonoids contained in a flavonoid-enriched fraction $(50 \mu \mathrm{g} / \mathrm{mL})$ extracted from the rhizomes of Sophora flavescens also showed in vitro inhibitory effects greater than $50 \%$ on the NO production [16]. Isorhamnetin, kaempferol, and quercetin inhibited $i$ NOS protein and $m$ RNA expression when tested in macrophages stimulated with lipopolysaccharide (LPS) [17]. Also, these compounds inhibited the activation of nuclear transcription factor $-\kappa \mathrm{B}(\mathrm{NF}-\kappa \mathrm{B})$.

In nature, flavonoids are generally attached to sugar residues that modify the mechanism of absorption and their ability to enter cells or to interact with transporters and cellular lipoproteins [18]. Therefore there are differences among the biological effects exerted by flavonoid glycosides according to the type, number, and position of sugar moieties $[19,20]$. de Melo et al. [21] showed that kaempferol 3-O- $\beta$ glucopyranoside-7-O- $\alpha$-rhamnopyranoside and kaempferol 7-O- $\alpha$-rhamnopyranoside inhibited the croton oil-induced ear edema by $46.5 \%$ and $33.3 \%$, respectively. Likewise, kaempferol 3-O- $\beta$-D-apiofuranosyl- $(1 \rightarrow 2)$ - $\alpha$-L-arabinofuranosyl-7-O- $\alpha$-L-rhamnopyranoside $(10 \mu \mathrm{M})$ showed better inhibitory effects on TNF- $\alpha$ and IL-12 production than kaempferol 3-O- $\beta$-D-apiofuranosy- $(1 \rightarrow 4)-\alpha$-L-rhamnopyranosyl-7-O- $\alpha$-L-rhamnopyranoside $(40 \mu \mathrm{M})$ [22].

Opuntia ficus-indica (OFI) has been used in Mexico as a food and as a remedy against different health disorders related to skin such as inflammation $[23,24]$. This plant is a natural source of flavonoids, mainly isorhamnetin glycosides [12-14, 25]. Isorhamnetin and isorhamnetin-3-O-galactoside possess in vitro anti-inflammatory activity [26, 27].

Considering all the above, the aim of this research work was to evaluate the in vitro and in vivo anti-inflammatory effects of an OFI extract and compare the bioactivity of different isorhamnetin glycosides isolated from this source. OFI extract and isolated isorhamnetin diglycosides and triglycosides were tested on RAW 264.7 macrophage cells in order to evaluate their effect on NO production. Later, these compounds were tested in a rat ear edema inflammation model to evaluate their effect on COX-2, TNF- $\alpha$ and, IL-6 levels.

\section{Materials and Methods}

2.1. Reagents. Dulbecco's Modified Eagle Medium: Nutrient Mixture F-12 (DMEM-F12), ampicillin/streptomycin, and phosphate-buffered saline $(\mathrm{pH}$ 7.4) were purchased from Gibco Invitrogen (Carlsbad, CA). Trypsin-EDTA (0.25\%) and fetal bovine serum were obtained from HyClone Thermo Scientific (Logan, UT). Triton X-100 was acquired from Research Organics (Cleveland, OH). Lipopolysaccharides (LPS) from Salmonella enterica serotype typhimurium L7261, as well as croton oil, indomethacin, isorhamnetin standard, and formic acid solution HPLC grade were purchased from Sigma-Aldrich (St. Louis, MO). Chromatography grade water and methanol (VWR International LLC, West Chester, PA) were used for high-pressure liquid chromatograph equipped with a photodiode array detector (HPLC-PDA) and liquid chromatograph/mass selective detector time-of-flight (LC/MSD TOF) analysis.

2.2. Obtaining O. ficus-indica Extract. The taxonomic identification of Opuntia ficus-indica was done by $\mathrm{Ph}$.D. Rigoberto E. Vázquez-Alvarado at the School of Agronomy of Universidad Autónoma de Nuevo León (UANL), México. OFI cladodes were harvested at 7 months and grown in the region of Montemorelos, Nuevo Leon, México, and then they were processed into powder according to Santos-Zea et al. [14]. OFI extract was obtained by alkaline hydrolysis following the method previously reported by Antunes-Ricardo et al. [12].

2.3. Identification, Quantification, and Purification of Isorhamnetin Glycosides in OFI Extract. Identification and quantification of isorhamnetin glycosides were performed according to the method described by Antunes-Ricardo et al. [12] using HPLC-PDA (Agilent 1100 Series Santa Clara, CA). Purification of isorhamnetin glycosides was done by semipreparative chromatography according to the method described by Antunes-Ricardo et al. [12] using a semipreparative Zorbax SB-C18 $(9.4 \times 250 \mathrm{~mm}, 5 \mu \mathrm{m})$ column at $17^{\circ} \mathrm{C}$, flow rate of $2.0 \mathrm{~mL} / \mathrm{min}$, and injection volume of $50 \mu \mathrm{L}$. Water with $0.1 \%$ formic acid (A) and methanol $80 \%$ (B) were used as mobile phases, starting with $35 \%$ of B and increasing to $60 \%$ and $90 \%$ after 2 and $19 \mathrm{~min}$, respectively, and then decreasing to $0 \% \mathrm{~B}$ for the next $3 \mathrm{~min}$. Percentages of purity of $90 \%$ or more were obtained for the isorhamnetin glycosides. LC/MSD TOF technology (Model G1969A Agilent 1100 Santa Clara, CA) was used to identify the isorhamnetin glycosides according to the method described by Santos-Zea et al. [14].

2.4. RAW 264.7 Cell Culture. RAW 264.7, a murine macrophage cell line, was obtained from the American Type Culture Collection (ATCC, Manassas, VA). Cells were cultured in petri plates with Dulbecco's Modified Eagle Medium (DMEM) supplemented with $10 \%$ fetal bovine serum (FBS) and $1 \%$ antibiotic and incubated at $37^{\circ} \mathrm{C}$ and $5 \%$ of $\mathrm{CO}_{2}$ (NuAire, Plymouth, MN). To evaluate the effects of an OFI extract and isolated isorhamnetin glycosides on nitric oxide production, cells were plated in 96-well plate $\left(5 \times 10^{4}\right.$ cells/well) and allowed to adhere for $4 \mathrm{~h}$. After that, different concentrations $(25,50$, or $125 \mathrm{ng}$ isorhamnetin equivalents (IsoEq/mL) of the compounds were added and incubated for another $24 \mathrm{~h}$. Following, half of wells were stimulated with LPS at $10 \mu \mathrm{g} / \mathrm{mL}$ (final concentration) while the other half were used as control for each sample.

2.5. Measurement of Nitrite Concentration. Production of nitric oxide (NO) was measured by nitrites determination, which is a stable and nonvolatile breakdown product of NO. After incubation with the different treatments, $100 \mu \mathrm{L}$ of supernatant was transferred to another 96-well plate to measure nitrites concentration using the Griess Reagent System (Promega, Madison, WI) at $550 \mathrm{~nm}$. Data was expressed as percentage of inhibition of NO production. The remaining 
cells and medium in original plate were used to measure cell viability.

2.6. Measurement of RAW 264.7 Cell Viability. Cell viability was determined by CellTiter 96 AQueous One Solution Cell Proliferation Assay (Promega, Madison, WI). Absorbance was measured at $490 \mathrm{~nm}$ with a 96-well microplate reader (Synergy HT, Bio-Tek, Winooski, VM). Cell viability was calculated by dividing the absorbance of cells treated by the absorbance of control cells, and this ratio was expressed as percentage.

2.7. Animals. Male Wistar rats (250-300 g) were purchased from Bioinvert (Mexico, DF, Mexico). Animals were divided into seven groups $(n=8)$ and kept in a room with controlled temperature $\left(25^{\circ} \mathrm{C}\right)$ and relative humidity $(50 \pm 5 \%)$ for $12 \mathrm{~h}$ light/dark cycles with food and water ad libitum. The Institutional Committee on Care and Use of Experimental Animals at the Tecnológico de Monterrey approved this study with the protocol number 2014-RE-001.

2.8. Acute Ear Edema Induced by Croton Oil. Experiments were carried out as described previously [28, 29]. Cutaneous inflammation was induced by the application of $5 \%$ croton oil $(20 \mu \mathrm{L})$ in acetone in the outer surface of the right ear in rats. The left ear received an equal volume of vehicle (acetone). After 30 minutes, OFI extract, isorhamnetin glycosides, isorhamnetin standard at dose of $1.0 \mu \mathrm{moL}$ IsoEq/ear, or indomethacin $(1.0 \mu \mathrm{moL} / \mathrm{ear})$ was applied topically in the right ear in the test groups. The left ear of each experimental subject of the test groups received an equal volume of the vehicle. The control group only received vehicle as treatment. Test compounds and vehicle were applied by an automatic micropipette. Animals were euthanized by cervical dislocation 4 hours after croton oil application. Both ears were removed with a metal punch (6 $\mathrm{mm}$ diameter disc) and weighed (APX-100, Denver Instrument, Denver, CO). Ear edema was calculated by subtracting the weight of the left ear (untreated) from the right ear (treated). The results were expressed as percentage of inhibition of ear edema with respect to the control group according to the following: inhibition (\%): [(ear edema of control group) - (ear edema of treated group) $] \times 100 /[$ ear edema of control group] .

2.9. Measurement of COX-2, TNF- $\alpha$, and IL-6 in the Ear Edema Model Induced by Croton Oil. Ear biopsy samples were pooled by group and homogenized vigorously (Tissue Tearor, BioSpec Products, Racine, WI) in $1.5 \mathrm{~mL}$ of extraction buffer (containing $10 \mathrm{mM}$ Tris $\mathrm{pH}$ 7.4, $150 \mathrm{mM} \mathrm{NaCl}$, and $1 \%$ Triton X-100) per gram of sample. The homogenates were centrifuged at $13,000 \mathrm{~g}$ for 10 minutes at $4^{\circ} \mathrm{C}$ in a Microfuge $22 \mathrm{R}$ centrifuge (Beckman Coulter, Atlanta, GA) and the supernatants were stored at $-80^{\circ} \mathrm{C}$ until being analyzed. The inhibition of COX-2 activity was determined by the COX Colorimetric Inhibitor Screening Assay (Cayman Chemical, Ann Arbor, MI). The levels of TNF- $\alpha$ and IL- 6 in the supernatants were measured using commercially available sandwich enzyme-linked immunosorbent assay (ELISA) kits

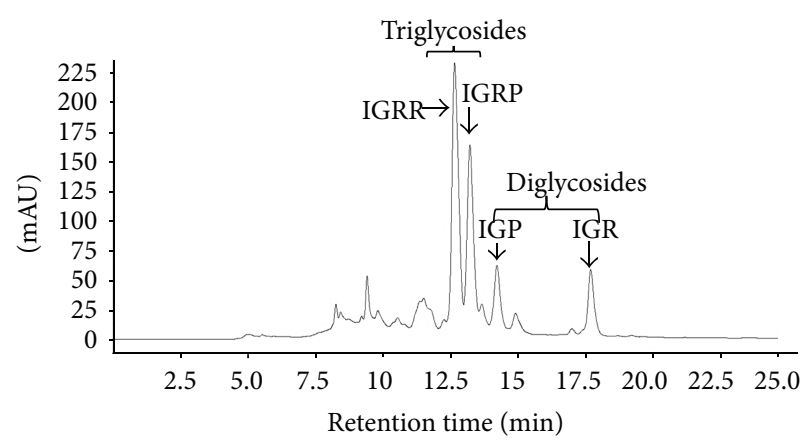

FIgURE 1: Chromatogram obtained at $365 \mathrm{~nm}$ showing the most abundant isorhamnetin glycosides found in Opuntia ficusindica extract. IGRR: isorhamnetin-glucosyl-rhamnosyl-rhamnoside; IGRP: isorhamnetin-glucosyl-rhamnosyl-pentoside; IGP: isorhamnetin-glucosyl-pentoside; IGR: isorhamnetin-glucosylrhamnoside.

(Invitrogen Corp., Camarillo, CA) according to the manufacturer's instructions. The results were expressed as percentage of inhibition with respect to control.

2.10. Statistical Analysis. All measurements were performed at least in triplicate and results were expressed as mean \pm standard deviation. Statistical analyses were performed with the JMP 8.0 software (SAS Institute Inc., Cary, NC). Data was analyzed by ANOVA methodology followed by Tukey's HSD tests with a significance level of $P<0.05$.

\section{Results}

3.1. Identification and Quantification of Isorhamnetin Glycoside in OFI Extract. The most abundant isorhamnetin diglycosides were isorhamnetin-glucosyl-pentoside (IGP) and isorhamnetin-glucosyl-rhamnoside (IGR) whereas isorhamnetin-glucosyl-rhamnosyl-rhamnoside (IGRR) and isorhamnetin-glucosyl-rhamnosyl-pentoside (IGRP) were the most abundant triglycosides (Figure 1). Table 1 shows quantification of isorhamnetin glycosides in the OFI extract and their identification by comparison with previous reports of lambda maxima (UV/VIS) obtained by HPLC and $\mathrm{m} / \mathrm{z}$ $[\mathrm{M}+\mathrm{H}]$ obtained by mass spectrometry (see Supplementary Figures S1 and S2 in Supplementary Material available online at http://dx.doi.org/10.1155/2015/847320). Along with the molecular ion, sodium adducts were obseved in each mass spectra. Ionization conditions allowed the detection of fragments generated by the loss of three, two and one sugar moieties observed in the triglycosides mass spectrum (Figure S1); likewise, fragments generated by the loss of two and one sugar moieties were observed in the diglycosides mass spectrum (Figure S2). The $m / z$ [317.05] corresponding to isorhamnetin aglycone appears in all mass spectrum.

3.2. Effect of OFI Extract and Isorhamnetin Glycosides in Cell Viability and NO Production in RAW 264.7 Macrophage Cells Stimulated by LPS. Isorhamnetin triglycosides, IGRR, and IGRP did not affect the cell viability at any of 
TABLE 1: Identification and quantification of most abundant isorhamnetin glycosides associated with Opuntia ficus-indica extract.

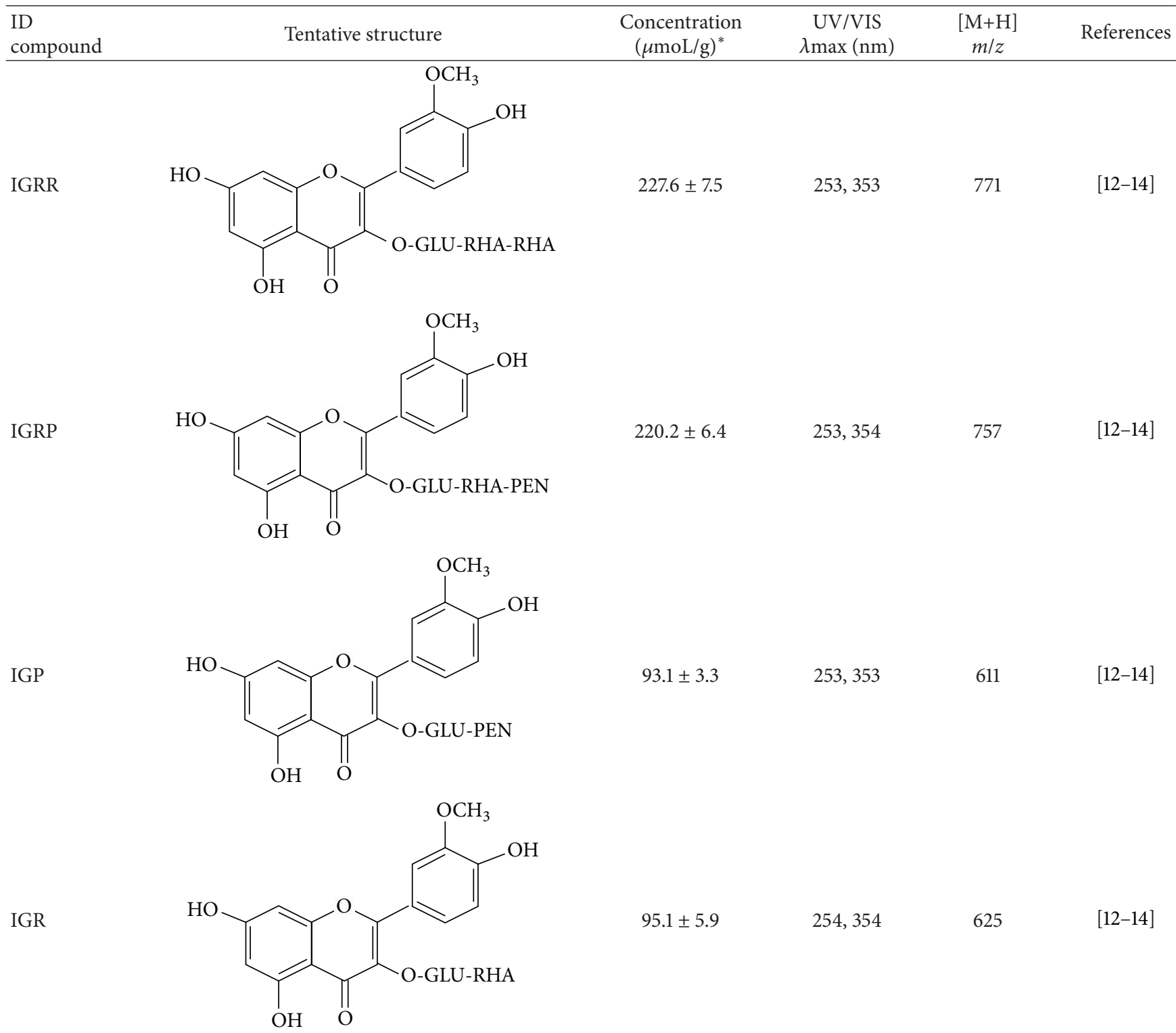

${ }^{*}$ Concentration expressed in $\mu \mathrm{moL}$ of IsoEq/g of OFI extract. IGRR: isorhamnetin-glucosyl-rhamnosyl-rhamnoside; IGRP: isorhamnetin-glucosylrhamnosyl-pentoside; IGP: isorhamnetin-glucosyl-pentoside; IGR: isorhamnetin-glucosyl-rhamnoside.

the concentrations tested (Table 2). In contrast, IGP, IGR, and OFI extract showed a slight decrease in cell viability $(84.9 \pm$ $4.0 \%, 81.6 \pm 3.0 \%$, and $89.9 \pm 1.9 \%$, resp.) when tested at $125 \mathrm{ng}$ IsoEq/mL.

Regarding the inhibitory effects of isorhamnetin glycosides and OFI extract on NO production (Table 3), data showed that OFI extract was more potent inhibitor than isorhamnetin at all tested concentrations, exhibiting $73.5 \pm$ $4.8 \%$ as the maximum inhibition value at $125 \mathrm{ng}$ IsoEq $/ \mathrm{mL}$.

At $25 \mathrm{ng}$ IsoEq/mL, IGRP and diglycosides IGP and IGR were better inhibiting NO production showing $53.2 \pm 4.7 \%$, $57.4 \pm 9.1 \%$, and $60.0 \pm 3.4 \%$ of inhibition, respectively. At the same concentration, IGR showed also better effect inhibiting NO production than OFI extract. But when the concentration was increased to 50 and $125 \mathrm{ng}$ IsoEq/mL, IGR and OFI extract increased NO inhibition by $35.9 \%$ and $32.7 \%$, respectively. Although isorhamnetin bioactivity significantly increased with concentration, it was still the least potent inhibitor of NO production $(47.6 \pm 5.8 \%)$.

3.3. Effect of Isorhamnetin Glycosides and OFI Extract in Acute Ear Edema Induced by Croton Oil. The diglycoside IGR and indomethacin showed the greatest potential to inhibit the ear edema induced by croton oil, $77.4 \pm 5.7 \%$ and $69.5 \pm$ $5.3 \%$, respectively (Figure 2). Inhibition exhibited by IGP was slightly lower $(65.3 \pm 5.9 \%)$ than the observed for IGR but similar to the observed for isorhamnetin. The isorhamnetin triglycoside IGRR showed lower inhibitory effect on ear inflammation $(44.7 \pm 8.2 \%)$ than IGR and OFI extract $(57.1 \pm$ 9.7\%). In this work isorhamnetin glycosides IGP and IGR, but 
TABLE 2: Effect of Opuntia ficus-indica (OFI) extract, isorhamnetin, and its glycosides in RAW 264.7 cell viability.

\begin{tabular}{lccc}
\hline Treatment & \multicolumn{3}{c}{ Cell viability $(\%)$} \\
\hline Isorhamnetin & $99.3 \pm 5.6^{\mathrm{a} *}$ & $50 \mathrm{ng} / \mathrm{mL}$ & $125 \mathrm{ng} / \mathrm{mL}$ \\
IGP & $96.7 \pm 2.4^{\mathrm{ab}}$ & $88.9 \pm 5.3^{\mathrm{a}}$ & $97.7 \pm 2.1^{\mathrm{a}}$ \\
IGR & $92.2 \pm 3.1^{\mathrm{b}}$ & $85.2 \pm 5.1^{\mathrm{b}}$ & $84.9 \pm 4.0^{\mathrm{cd}}$ \\
IGRR & $99.4 \pm 2.9^{\mathrm{a}}$ & $98.5 \pm 2.3^{\mathrm{a}}$ & $95.7 \pm 4.0^{\mathrm{ab}}$ \\
IGRP & $99.4 \pm 3.9^{\mathrm{a}}$ & $97.5 \pm 2.8^{\mathrm{a}}$ & $94.2 \pm 4.0^{\mathrm{ab}}$ \\
OFI extract & $94.5 \pm 1.2^{\mathrm{ab}}$ & $92.6 \pm 4.8^{\mathrm{ab}}$ & $89.9 \pm 1.9^{\mathrm{bc}}$ \\
\hline
\end{tabular}

${ }^{\#}$ Concentration expressed in ng IsoEq/mL. IGP: isorhamnetin-glucosylpentoside; IGR: isorhamnetin-glucosyl-rhamnoside; IGRR: isorhamnetinglucosyl-rhamnosyl-rhamnoside; IGRP: isorhamnetin-glucosyl-rhamnosylpentoside. ${ }^{*}$ Different letters in each column denote significant differences among treatments $(P<0.05)$.

TABLE 3: Percentage of inhibition of nitric oxide production in RAW 264.7 cells incubated with different concentrations of Opuntia ficusindica (OFI) extract, isorhamnetin, and its glycosides.

\begin{tabular}{lccc}
\hline \multirow{2}{*}{ Treatment } & \multicolumn{3}{c}{$\begin{array}{c}\text { Nitric oxide inhibition (\%) } \\
\text { Concentration (ng IsoEq/mL) }\end{array}$} \\
& 25 & 50 & 125 \\
\hline Isorhamnetin & $19.6 \pm 2.2^{\mathrm{Cd}}$ & $47.6 \pm 5.8^{\mathrm{Bc}}$ & $55.5 \pm 3.1^{\mathrm{Ac}}$ \\
IGP & $57.4 \pm 9.1^{\mathrm{Aab}}$ & $58.7 \pm 4.2^{\mathrm{Aab}}$ & $60.7 \pm 4.3^{\mathrm{Abc}}$ \\
IGR & $60.0 \pm 3.4^{\mathrm{Ba}}$ & $65.4 \pm 4.2^{\mathrm{ABa}}$ & $68.7 \pm 5.0^{\mathrm{Aab}}$ \\
IGRR & $50.6 \pm 4.0^{\mathrm{Bb}}$ & $54.2 \pm 2.8^{\mathrm{Bbc}}$ & $61.2 \pm 5.8^{\mathrm{Abc}}$ \\
IGRP & $53.2 \pm 4.7^{\mathrm{Aab}}$ & $54.4 \pm 6.3^{\mathrm{Abc}}$ & $59.9 \pm 4.1^{\mathrm{Ac}}$ \\
OFI extract & $40.8 \pm 4.5^{\mathrm{Cc}}$ & $57.2 \pm 5.7^{\mathrm{Bab}}$ & $73.5 \pm 4.8^{\mathrm{Aa}}$ \\
\hline
\end{tabular}

IGP: isorhamnetin-glucosyl-pentoside; IGR: isorhamnetin-glucosyl-rhamnoside; IGRR: isorhamnetin-glucosyl-rhamnosyl-rhamnoside; IGRP: isorhamnetin-glucosyl-rhamnosyl-pentoside.

a,b,c Different lower case letters denote significant differences among treatments.

${ }_{A, B, C}$ Different upper case letters denote significant differences among concentrations $(P<0.05)$.

not IGRR, showed better topical anti-inflammatory potential than OFI extract.

3.4. Effect of Isorhamnetin Glycosides and OFI Extract in Proinflammatory COX-2 Activity and TNF- $\alpha$ and IL-6 Production. Proinflammatory cytokines TNF- $\alpha$ and IL-6 were determined in order to clarify the topical anti-inflammatory mechanisms of isorhamnetin glycosides present and isolated from the OFI extract (Figure 3). The greatest suppression of TNF- $\alpha$ production was observed in ears treated with IGR $(88.3 \pm 0.5 \%)$ while the OFI extract showed greater IL-6 suppression $(59.1 \pm 1.4 \%)$. The pure IGR increased TNF- $\alpha$ inhibition in $30.9 \%$ in contrast to the inhibition caused by the OFI crude extract. The OFI extract showed more effect as an inhibitor of COX-2 $(74.8 \pm 2.9 \%)$ than indomethacin and IGR which exerted the same inhibitory effect $(70.9 \pm 0.9 \%$ and $68.3 \pm 0.6 \%$, resp.).

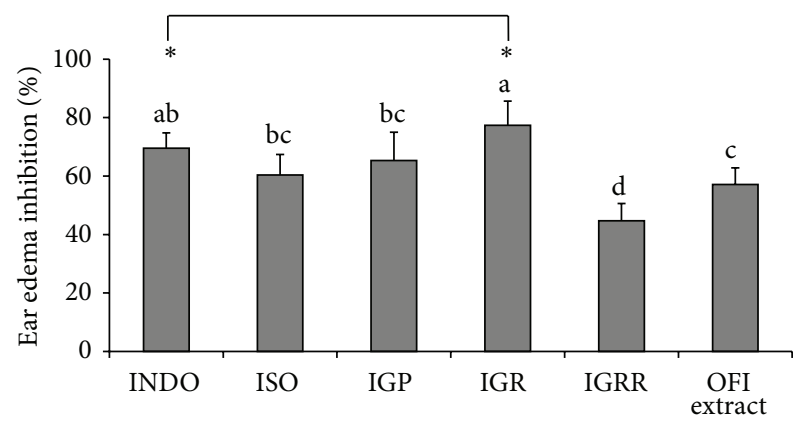

FIGURE 2: Effect of indomethacin (INDO), isorhamnetin (ISO), isorhamnetin glycosides, and Opuntia ficus-indica (OFI) extract on croton oil-induced rat ear edema. IGP: isorhamnetin-glucosylpentoside; IGR: isorhamnetin-glucosyl-rhamnoside; IGRR: isorhamnetin-glucosyl-rhamnosyl-rhamnoside. ${ }^{\mathrm{a}, \mathrm{b}, \mathrm{c}, \mathrm{d}}$ Different letters denote significant differences among treatments $(P<0.05)$. ${ }^{*}$ Treatment with highest potential to inhibit ear edema.

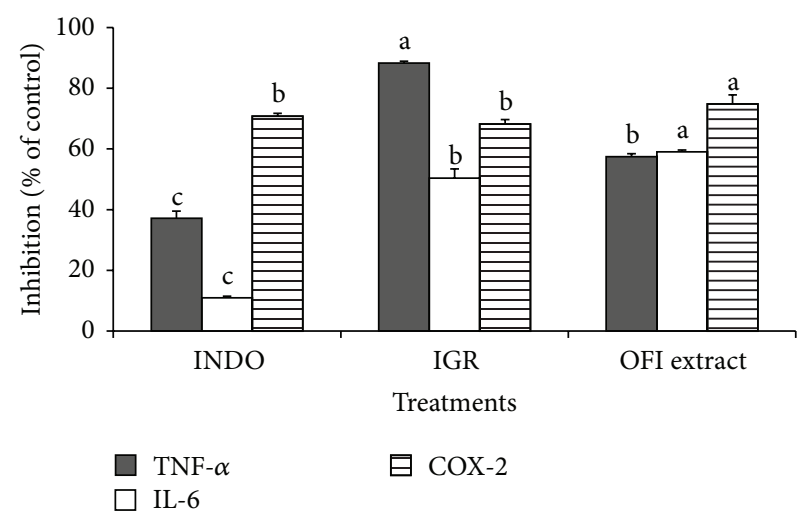

FIGURE 3: Effect of indomethacin (INDO), isorhamnetin-glucosylrhamnoside (IGR), and Opuntia ficus-indica (OFI) extract on TNF$\alpha$ and interleukin- (IL-) 6 levels and on COX-2 activity in rat ears tissue homogenate. Indomethacin was used as positive control. Control group only received croton oil $5 \% \mathrm{v} / \mathrm{v}$ as irritant agent. Data represent means \pm standard deviations of at least 6 replicates. ${ }^{\mathrm{a}, \mathrm{b}, \mathrm{c}}$ Different letters denote significant differences among treatments $(P<0.05)$.

\section{Discussion}

The observed effects on cell viability were similar to those reported by Benayad et al. [23] who tested the anti-inflammatory activities of extracts from Moroccan Opuntia ficus-indica flowers. Cell viability of macrophage RAW 264.7 cells was $\geq 90 \%$ for all samples at the concentrations tested [23]. Therefore, reduction of NO was not due to the cytotoxicity of isorhamnetin glycosides since cell viability was above $80 \%$.

All isolated isorhamnetin glycosides showed inhibitory effects higher than $50 \%$ on NO production even at concentrations as low as $25 \mathrm{ng}$ IsoEq/mL. Isorhamnetin diglycosides had a higher anti-inflammatory potential than triglycosides. Other reports had demonstrated that kaempferol glycosides inhibited differently the NO production. Particularly, 
kaempferol 7-O- $\alpha$-L-rhamnopyranoside showed an IC50 value of $41.2 \mu \mathrm{M}$, whereas kaempferol triglycosides and diglycosides at the dosage of $100 \mu \mathrm{M}$ had an inhibitory effect of less than 10\% [30]. Fang et al. [22] showed that kaempferitrin and kaempferol-3-O- $\beta$-D-apiofuranosyl(1-2)- $\alpha$-L-arabinofuranosyl-7-O- $\alpha$-L-rhamnopyranoside differently inhibited the NO production exhibiting an IC50 of $40 \mu \mathrm{M}$ and $15 \mu \mathrm{M}$, respectively.

Additionally, Rho et al. [31] demonstrated that the position and the number of sugar moieties affect the suppression of NO production exerted by flavonol glycosides. Kaempferol 7-O- $\alpha$-L-rhamnopyranoside showed more potential to inhibit NO production (IC50 $=37.7 \mu \mathrm{M})$ than kaempferol 3-O- $\alpha$-L-rhamnopyranoside (IC50 > $100 \mu \mathrm{M}$ ). Likewise, Rho et al. [31] showed that the presence of an additional rhamnose moiety at the 3-position caused a loss in $\mathrm{NO}$ inhibitory activity as in the case of kaempferol-3, 7-O- $\alpha$ dirhamnoside (IC50>100 $\mu \mathrm{M})$. These results are consistent with our findings where the diglycoside IGR showed greater inhibitory activity on NO production compared with its corresponding triglycoside IGRR that has an additional rhamnose moiety.

Isorhamnetin glycosides had a higher inhibition on nitric oxide production compared to aglycone tested at $25 \mathrm{ng}$ IsoEq/mL (Table 3). In contrast, previous reports indicated that a quercetin glycoside had a similar effect to the aglycone only when tested at a concentration 30 times higher [32]. But baicalein and baicalein-6- $\alpha$-glucoside when they were tested at $40 \mu \mathrm{M}$ showed similar inhibitory activities on the NO production (approximately 60\%) [33].

The effect of OFI extract at $50 \mathrm{ng}$ IsoEq/mL was comparable with the $\mathrm{NO}$ production inhibition induced by an extract of $O$. ficus-indica flowers ( $0.5 \mathrm{mg}$ extract $/ \mathrm{mL}$ ) in RAW 264.7 cell line (>60\%) [23]. Isolated isorhamnetin glycosides showed greater NO production inhibition than OFI extract when tested at $25 \mathrm{ng}$ IsoEq/mL. But, when concentration was increased to $125 \mathrm{ng}$ IsoEq/mL, OFI extract inhibited the NO production similarly or more potently than the isorhamnetin glycosides tested. Kazłowska et al. [34] reported that Porphyra dentata crude extract was more active inhibiting NO production compared to its pure compounds (catechol, rutin, and hesperidin). Harasstani et al. [35] have demonstrated that when flavonoids are combined, as occurs in plant extracts, these can significantly increase their inhibitory effect upon NO production.

In the ear edema inflammation model, IGR and indomethacin showed the better effects. The effect of IGR was comparable with the $81 \%$ of inhibition induced by the tiliroside (flavonol acyl-glucoside) on an acute TPA-induced ear edema inflammation model [36]. As observed in the in vitro experiments, triglycoside IGRR showed lower anti-inflammatory effects compared with diglycosides and indomethacin. The difference in hydrophobicity between isorhamnetin diglycosides and triglycosides alters the cellmembrane permeability, which may be the reason for the better effect exerted by diglycosides. It has been suggested that the compounds with a MW $<500 \mathrm{Da}$ are suitably transported across skin $[37,38]$.
Different anti-inflammatory effects were observed among isorhamnetin diglycosides, IGR and IGP, although they only differed in the pentose methylation. These results indicated that the type of sugar affected bioactivity, contrary to those reported by Sosa et al. [39] who showed that quercetin-3$\mathrm{O}$-galactoside and quercetin-3-O-glucoside at $1.0 \mu \mathrm{moL} /$ ear exhibited similar inhibition in a mouse ear edema induced by croton oil ( $52 \%$ and $51 \%$, resp.). Erdemoglu et al. [40] demonstrated that quercetin-3-O-galactoside and quercetin3-O-rhamnoside, tested at $0.5 \mathrm{mg} /$ ear, inhibited differently the edema induced in mice by TPA ( $32 \%$ and $44.7 \%$, resp.), suggesting that the presence of rhamnose could increase the anti-inflammatory potential.

Although some reports indicated that flavonoid glycosylation reduced the anti-inflammatory effect, isorhamnetin (ISO) and IGP showed similar effects on the rat ear edema inflammation model. This trend had been previously reported for hesperetin and the hesperetin-7-rutinoside that had similar anti-inflammatory effects ( $44 \%$ and $45 \%$, resp.) in mice model of ear swelling induced by xylene [41].

The inhibitory effect of the OFI extract on ear edema was comparable to that produced by the chloroform extract from Senna villosa leaves (57.9\%) in the TPA-induced ear edema model and slightly greater (52.4\%) than the extract of Pistacia khinjuk L. leaves, which also was rich in glycosylated flavonoids [42, 43]. Some isolated compounds can be more potent anti-inflammatory drugs than their source extract. Mo et al. [28] reported that the $\mathrm{MeOH}$ extracts of Lithraea molleoides (Vell.) Engl. (Anacardiaceae) inhibited the ear edema induced by TPA by $21 \%$, while the methyl gallate, one of the main compounds in $\mathrm{MeOH}$ extract, at an equivalent concentration, produced $63 \%$ of ear edema inhibition. Likewise, an ethanolic extract of Myagropsis myagroides (90 $\mu \mathrm{g} /$ ear) markedly inhibited the phorbol myristate acetate(PMA-) induced ear edema with 66.8\% inhibition $(P<$ $0.05)$, one-third of $6,6^{\prime}$-bieckol activity which was tested at $30 \mu \mathrm{g} /$ ear [44]. In this investigation, although the OFI extract showed an adequate inhibitory effect on ear edema, it did not exceed the effects observed with isorhamnetin diglycosides IGR and IGP. Based on these results, the order of antiinflammatory potential against the ear edema was IGR = INDO $>$ IGP $=$ ISO $>$ OFI extract $>$ IGRR.

Tumor necrosis factor (TNF) is a potent mediator of inflammation with a powerful proinflammatory capacity, especially within immune cells capable of producing a cascade of downstream cytokines and chemokines expressed by monocytes/macrophages [45]. Meanwhile, IL-6 is an important factor for the synthesis of acute phase proteins, whose serum level is increased in acute and chronic inflammatory diseases such as cancer [46, 47]. In skin inflammation, cytokines such as TNF- $\alpha$ and IL- 6 from resident cells keratinocytes bind to receptors on the vascular endothelium, activating cellular signaling pathways and inducing expression of vascular endothelial cell adhesion molecules [48].

Both IGR and OFI extract inhibited COX-2 activity and inflammatory cytokines IL- 6 and TNF- $\alpha$. TNF- $\alpha$ activation stimulates the IL- 6 synthesis in several cell types and, in turn, IL-6 inhibits the TNF- $\alpha$ production providing a negative feedback, thus limiting the acute inflammatory response [49]. 
IGR showed more pronounced effect on TNF- $\alpha$ than IL6 or COX-2 activity, indicating that its anti-inflammatory effect was mediated by the inhibition of proinflammatory cytokines. Differences in the cytokine modulation indicate that at least a part of the IL- 6 production could be directed and not mediated by TNF- $\alpha$ activation [50]. Mueller et al. [51] observed that an apple extract containing 5\% quercetin and $30 \%$ phloridzin (glycosylated phenolic compound) suppressed more potently the TNF- $\alpha$ production (89\%) than that of IL-6 (32\%) in a LPS-stimulated macrophage model.

The OFI extract showed greater inhibition on COX-2 activity than on TNF- $\alpha$ or IL-6. Inhibitory effects of OFI extract on TNF- $\alpha$ and IL- 6 were similar to those observed for quercetin $(59.1 \pm 3.3 \%$ and $57.9 \pm 9.9 \%$, resp.) measured in human blood [52].

On the other hand, Lee et al. [53] proved that a flavonol-rich hexane fraction from Rhus verniciflua stokes and its major compound fisetin affected differently the TNF$\alpha$ and IL-6 production in vitro. The $R$. verniciflua hexane fraction $(1 \mu \mathrm{g} / \mathrm{mL})$, which contained about $0.0827 \mu \mathrm{g} / \mathrm{mL}$ of fisetin, suppressed approximately $26.3 \%$ of the TNF- $\alpha$ production in ear edema. When pure fisetin was tested at $0.1 \mu \mathrm{g} / \mathrm{mL}$, the TNF- $\alpha$ inhibition increased. Likewise, a crude hydroalcoholic extract of Croton antisyphiliticus Mart. and its isolated glycosylated flavonoid (apigenin- $\beta-D-$ glucopyranosyl) showed similar suppressing effect on TNF$\alpha$ production despite the differences among the concentrations tested (100 mg/Kg and $10 \mathrm{mg} / \mathrm{Kg}$, resp.) [54]. Results presented herein agree with previous reports for glycosylated flavonoids such as the tiliroside, wogonoside, kaempferol3-O-glucoside, quercetin 3-O-glucoside, and isorhamnetin3-O-glucoside as important inhibitors of TNF- $\alpha$ and IL-6 production [55-58].

Anti-inflammatory effects of flavonoids can occur through different molecular mechanisms such as inhibition of histamine release from basophils, inhibition of proinflammatory eicosanoids production, or by inhibiting the production of proinflammatory cytokines [23]. In this study it was demonstrated that isorhamnetin glycosides and the OFI extract modulate the inflammatory response by suppressing the COX-2 activity and also by the reduction in levels of NO, IL-6, and TNF- $\alpha$ both in vitro and in vivo.

\section{Conclusions}

The Opuntia ficus-indica (OFI) extract and its isorhamnetin glycosides exerted anti-inflammatory effects. In vitro, isorhamnetin diglycoside IGR and the OFI extract significantly reduced the nitric oxide production in RAW 264.7 macrophage cells without significantly affecting viability, while, in vivo, IGR showed an efficacy comparable to indomethacin to reduce rat ear edema induced by croton oil.

The attached sugar moiety affected the in vivo antiinflammatory effects of the diglycosides IGP and IGR. Likewise, the glycosylation degree affected the bioactivity, the triglycoside IGRR being less bioactive compared to the diglycoside IGR. The topical anti-inflammatory effect of IGR was mediated by the suppression of COX-2 activity and inhibition on the production of proinflammatory cytokines such as TNF- $\alpha$ and IL- 6 .

Further research is needed in order to determine the permeability and absorption of these compounds and evaluate their efficacy in dermatological preparations. Additional experiments are required to validate the mechanism of action, including iNOS expression and other genes involved in the inflammatory response.

\section{Abbreviations}

IsoEq: Isorhamnetin equivalents

OFI: Opuntia ficus-indica.

\section{Conflict of Interests}

The authors declare that there is no conflict of interests regarding the publication of this paper.

\section{Acknowledgments}

The authors acknowledge the financial support from the Consejo Nacional de Ciencia y Tecnología (CONACYT-CB Research Project 1168708 and Scholarship Program) and the Tecnológico de Monterrey (Nutriomics Research Group). Also they acknowledge the donation of Opuntia ficus-indica powder by Alimentos Funcionales S. de R.L.M.I.

\section{References}

[1] D. R. Bickers and M. Athar, "Oxidative stress in the pathogenesis of skin disease," Journal of Investigative Dermatology, vol. 126, no. 12, pp. 2565-2575, 2006.

[2] E. Omori, S. Morioka, K. Matsumoto, and J. Ninomiya-Tsuji, "TAK1 regulates reactive oxygen species and cell death in keratinocytes, which is essential for skin integrity," The Journal of Biological Chemistry, vol. 283, no. 38, pp. 26161-26168, 2008.

[3] S. Reuter, S. C. Gupta, M. M. Chaturvedi, and B. B. Aggarwal, "Oxidative stress, inflammation, and cancer: how are they linked?" Free Radical Biology and Medicine, vol. 49, no. 11, pp. 1603-1616, 2010.

[4] C. N. Young, J. I. Koepke, L. J. Terlecky, M. S. Borkin, S. L. Boyd, and S. R. Terlecky, "Reactive oxygen species in tumor necrosis factor- $\alpha$-activated primary human keratinocytes: implications for psoriasis and inflammatory skin disease," Journal of Investigative Dermatology, vol. 128, no. 11, pp. 2606-2614, 2008.

[5] A. Chiricozzi, E. Guttman-Yassky, M. Suárez-Farĩas et al., "Integrative responses to IL-17 and TNF- $\alpha$ in human keratinocytes account for key inflammatory pathogenic circuits in psoriasis," Journal of Investigative Dermatology, vol. 131, no. 3, pp. 677-687, 2011.

[6] W.-W. Li, T.-Z. Guo, X.-Q. Li, W. S. Kingery, and J. D. Clark, "Fracture induces keratinocyte activation, proliferation, and expression of pro-nociceptive inflammatory mediators," Pain, vol. 151, no. 3, pp. 843-852, 2010.

[7] M. Eddouks, D. Chattopadhyay, and N. A. Zeggwagh, "Animal models as tools to investigate antidiabetic and antiinflammatory plants," Evidence-Based Complementary and Alternative Medicine, vol. 2012, Article ID 142087, 14 pages, 2012. 
[8] D. Y. Lee, G. Choi, T. Yoon, M. S. Cheon, B. K. Choo, and H. K. Kim, "Anti-inflammatory activity of Chrysanthemum indicum extract in acute and chronic cutaneous inflammation," Journal of Ethnopharmacology, vol. 123, no. 1, pp. 149-154, 2009.

[9] S.-B. Yoon, Y.-J. Lee, S. K. Park et al., "Anti-inflammatory effects of Scutellaria baicalensis water extract on LPS-activated RAW 264.7 macrophages," Journal of Ethnopharmacology, vol. 125, no. 2, pp. 286-290, 2009.

[10] E. Fasano, S. Serini, N. Mondella et al., "Antioxidant and anti-inflammatory effects of selected natural compounds contained in a dietary supplement on two human immortalized keratinocyte lines," BioMed Research International, vol. 2014, Article ID 327452, 11 pages, 2014.

[11] H. A. S. Favacho, B. R. Oliveira, K. C. Santos et al., "Antiinflammatory and antinociceptive activities of Euterpe oleracea Mart., Arecaceae oil," Revista Brasileira de Farmacognosia, vol. 21, no. 1, pp. 105-114, 2011.

[12] M. Antunes-Ricardo, B. E. Moreno-García, J. A. GutiérrezUribe, D. Aráiz-Hernández, M. M. Alvarez, and S. O. SernaSaldivar, "Induction of apoptosis in colon cancer cells treated with isorhamnetin glycosides from Opuntia ficus-indica pads," Plant Foods for Human Nutrition, vol. 69, no. 4, pp. 331-336, 2014.

[13] G. Ginestra, M. L. Parker, R. N. Bennett et al., "Anatomical, chemical, and biochemical characterization of cladodes from prickly pear [Opuntia ficus-indica (L.) Mill.]," Journal of Agricultural and Food Chemistry, vol. 57, no. 21, pp. 10323-10330, 2009.

[14] L. Santos-Zea, J. A. Gutiérrez-Uribe, and S. O. Serna-Saldivar, "Comparative analyses of total phenols, antioxidant activity, and flavonol glycoside profile of cladode flours from different varieties of Opuntia spp," Journal of Agricultural and Food Chemistry, vol. 59, no. 13, pp. 7054-7061, 2011.

[15] S. Aquila, R. M. Giner, M. C. Recio, E. D. Spegazzini, and J. L. Ríos, "Anti-inflammatory activity of flavonoids from Cayaponia tayuya roots," Journal of Ethnopharmacology, vol. 121, no. 2, pp. 333-337, 2009.

[16] J. H. Jin, J. S. Kim, S. S. Kang, K. H. Son, H. W. Chang, and H. P. Kim, "Anti-inflammatory and anti-arthritic activity of total flavonoids of the roots of Sophora flavescens," Journal of Ethnopharmacology, vol. 127, no. 3, pp. 589-595, 2010.

[17] M. Hämäläinen, R. Nieminen, P. Vuorela, M. Heinonen, and E. Moilanen, "Anti-inflammatory effects of flavonoids: genistein, kaempferol, quercetin, and daidzein inhibit STAT-1 and NF$\kappa \mathrm{B}$ activations, whereas flavone, isorhamnetin, naringenin, and pelargonidin inhibit only NF- $\kappa \mathrm{B}$ activation along with their inhibitory effect on iNOS expression and NO production in activated macrophages," Mediators of Inflammation, vol. 2007, Article ID 45673, 10 pages, 2007.

[18] A. J. Day, J. M. Gee, M. S. DuPont, I. T. Johnson, and G. Williamson, "Absorption of quercetin-3-glucoside and quercetin-4/-glucoside in the rat small intestine: the role of lactase phlorizin hydrolase and the sodium-dependent glucose transporter," Biochemical Pharmacology, vol. 65, no. 7, pp. 11991206, 2003.

[19] G. M. Huber, H. P. Vasantha Rupasinghe, and F. Shahidi, "Inhibition of oxidation of omega-3 polyunsaturated fatty acids and fish oil by quercetin glycosides," Food Chemistry, vol. 117, no. 2, pp. 290-295, 2009.

[20] C.-T. Yen, P.-W. Hsieh, T.-L. Hwang, Y.-H. Lan, F.-R. Chang, and W. U. Yang-Chang, "Flavonol glycosides from Muehlenbeckia platyclada and their anti-inflammatory activity," Chemical \& Pharmaceutical Bulletin, vol. 57, no. 3, pp. 280-282, 2009.
[21] G. O. de Melo, D. D. C. Malvar, F. A. Vanderlinde et al., "Antinociceptive and anti-inflammatory kaempferol glycosides from Sedum dendroideum," Journal of Ethnopharmacology, vol. 124, no. 2, pp. 228-232, 2009.

[22] S.-H. Fang, Y. K. Rao, and Y.-M. Tzeng, "Inhibitory effects of flavonol glycosides from Cinnamomum osmophloeum on inflammatory mediators in LPS/IFN- $\gamma$-activated murine macrophages," Bioorganic \& Medicinal Chemistry, vol. 13, no. 7, pp. 2381-2388, 2005.

[23] Z. Benayad, C. Martinez-Villaluenga, J. Frias, C. GomezCordoves, and N. E. Es-Saf, "Phenolic composition, antioxidant and anti-inflammatory activities of extracts from Moroccan Opuntia ficus-indica flowers obtained by different extraction methods," Industrial Crops and Products, vol. 62, pp. 412-420, 2014.

[24] D.-W. Cho, D.-E. Kim, D.-H. Lee et al., "Metabolite profiling of enzymatically hydrolyzed and fermented forms of Opuntia ficus-indica and their effect on UVB-induced skin photoaging," Archives of Pharmacal Research, vol. 37, no. 9, pp. 1159-1168, 2014.

[25] T. E. Moussa-Ayoub, E. S. A. A. El-Hady, H. T. Omran, S. K. El-Samahy, L. W. Kroh, and S. Rohn, "Influence of cultivar and origin on the flavonol profile of fruits and cladodes from cactus Opuntia ficus-indica," Food Research International, vol. 64, pp. 864-872, 2014.

[26] B. Kim, Y.-E. Choi, and H.-S. Kim, "Eruca sativa and its flavonoid components, quercetin and isorhamnetin, improve skin barrier function by activation of peroxisome proliferatoractivated receptor (PPAR)- $\alpha$ and suppression of inflammatory cytokines," Phytotherapy Research, vol. 28, pp. 1359-1366, 2014.

[27] T. H. Kim, S.-K. Ku, and J.-S. Bae, "Anti-inflammatory activities of isorhamnetin-3-O-galactoside against HMGB1-induced inflammatory responses in both HUVECs and CLP-induced septic mice," Journal of Cellular Biochemistry, vol. 114, no. 2, pp. 336-345, 2013.

[28] J. Mo, P. Panichayupakaranant, N. Kaewnopparat, A. Nitiruangjaras, and W. Reanmongkol, "Topical anti-inflammatory and analgesic activities of standardized pomegranate rind extract in comparison with its marker compound ellagic acid in vivo," Journal of Ethnopharmacology, vol. 148, no. 3, pp. 901-908, 2013.

[29] L. H. Jones, D. S. P. Abdalla, and J. C. Freitas, "Effects of indole3 -acetic acid on croton oil- and arachidonic acid-induced mouse ear edema," Inflammation Research, vol. 44, no. 9, pp. 372-375, 1995.

[30] H.-Y. Lin and S.-T. Chang, "Kaempferol glycosides from the twigs of Cinnamomum osmophloeum and their nitric oxide production inhibitory activities," Carbohydrate Research, vol. 364, pp. 49-53, 2012.

[31] H. S. Rho, A. K. Ghimeray, D. S. Yoo et al., "Kaempferol and kaempferol rhamnosides with depigmenting and antiinflammatory properties," Molecules, vol. 16, no. 4, pp. 33383344, 2011.

[32] V. A. Vo, J.-W. Lee, J.-E. Chang et al., "Avicularin inhibits lipopolysaccharide-induced inflammatory response by suppressing ERK phosphorylation in RAW 264.7 macrophages," Biomolecules \& Therapeutics (Seoul), vol. 20, no. 6, pp. 532-537, 2012.

[33] K. H. Kim, Y. D. Park, H. Park et al., "Synthesis and biological evaluation of a novel baicalein glycoside as an antiinflammatory agent," European Journal of Pharmacology, vol. 744, pp. 147-156, 2014. 
[34] K. Kazłowska, T. Hsu, C.-C. Hou, W.-C. Yang, and G.-J. Tsai, "Anti-inflammatory properties of phenolic compounds and crude extract from Porphyra dentata," Journal of Ethnopharmacology, vol. 128, no. 1, pp. 123-130, 2010.

[35] O. A. Harasstani, S. Moin, C. L. Tham et al., "Flavonoid combinations cause synergistic inhibition of proinflammatory mediator secretion from lipopolysaccharide-induced RAW 264.7 cells," Inflammation Research, vol. 59, no. 9, pp. 711-721, 2010.

[36] A. Sala, M. C. Recio, G. R. Schinella et al., "Assessment of the anti-inflammatory activity and free radical scavenger activity of tiliroside," European Journal of Pharmacology, vol. 461, no. 1, pp. 53-61, 2003.

[37] C.-F. Lin, Y.-L. Leu, S. A. Al-Suwayeh, M.-C. Ku, T.-L. Hwang, and J.-Y. Fang, "Anti-inflammatory activity and percutaneous absorption of quercetin and its polymethoxylated compound and glycosides: the relationships to chemical structures," European Journal of Pharmaceutical Sciences, vol. 47, no. 5, pp. 857864, 2012.

[38] B. C. Finnin and T. M. Morgan, "Transdermal penetration enhancers: applications, limitations, and potential," Journal of Pharmaceutical Sciences, vol. 88, no. 10, pp. 955-958, 1999.

[39] S. Sosa, R. Pace, A. Bornancin et al., "Topical anti-inflammatory activity of extracts and compounds from Hypericum perforatum L.," Journal of Pharmacy and Pharmacology, vol. 59, no. 5, pp. 703-709, 2007.

[40] N. Erdemoglu, E. K. Akkol, E. Yesilada, and I. Caliş, "Bioassayguided isolation of anti-inflammatory and antinociceptive principles from a folk remedy, Rhododendron ponticum L. leaves," Journal of Ethnopharmacology, vol. 119, no. 1, pp. 172-178, 2008.

[41] A. E. Rotelli, T. Guardia, A. O. Juárez, N. E. de La Rocha, and L. E. Pelzer, "Comparative study of flavonoids in experimental models of inflammation," Pharmacological Research, vol. 48, no. 6, pp. 601-606, 2003.

[42] A. D. C. Susunaga-Notario, S. Pérez-Gutiérrez, M. A. ZavalaSánchez et al., "Bioassay-guided chemical study of the antiinflammatory effect of Senna villosa (Miller) HS Irwin \& Barneby (Leguminosae) in TPA-induced ear edema," Molecules, vol. 19, no. 7, pp. 10261-10278, 2014.

[43] A. Esmat, F. A. Al-Abbasi, M. M. Algandaby et al., "Antiinflammatory activity of Pistacia khinjuk in different experimental models: Isolation and characterization of its flavonoids and galloylated sugars," Journal of Medicinal Food, vol. 15, no. 3, pp. 278-287, 2012.

[44] E.-J. Joung, M.-S. Lee, J.-W. Choi et al., "Anti-inflammatory effect of ethanolic extract from Myagropsis myagroides on murine macrophages and mouse ear edema," BMC Complementary and Alternative Medicine, vol. 12, article 171, 2012.

[45] L. M. Sedger and M. F. McDermott, "TNF and TNF-receptors: from mediators of cell death and inflammation to therapeutic giants-past, present and future," Cytokine \& Growth Factor Reviews, vol. 25, no. 4, pp. 453-472, 2014.

[46] M. F. Neurath and S. Finotto, "IL-6 signaling in autoimmunity, chronic inflammation and inflammation-associated cancer," Cytokine \& Growth Factor Reviews, vol. 22, no. 2, pp. 83-89, 2011.

[47] K. Taniguchi and M. Karin, "IL-6 and related cytokines as the critical lynchpins between inflammation and cancer," Seminars in Immunology, vol. 26, no. 1, pp. 54-74, 2014.

[48] J. Y. Ahn, S. E. Choi, M. S. Jeong et al., "Effect of taxifolin glycoside on atopic dermatitis-like skin lesions in NC/Nga mice," Phytotherapy Research, vol. 24, no. 7, pp. 1071-1077, 2010.
[49] C. A. Feghali and T. M. Wright, "Cytokines in acute and chronic inflammation.," Frontiers in Bioscience, vol. 2, pp. d12-d26, 1997.

[50] M. Sironi, M. Gadina, M. Kankova et al., "Differential sensitivity of in vivo TNF and IL-6 production to modulation by anti-inflammatory drugs in mice," International Journal of Immunopharmacology, vol. 14, no. 6, pp. 1045-1050, 1992.

[51] M. Mueller, S. Hobiger, and A. Jungbauer, "Anti-inflammatory activity of extracts from fruits, herbs and spices," Food Chemistry, vol. 122, no. 4, pp. 987-996, 2010.

[52] D. Ribeiro, M. Freitas, S. M. Tomé et al., "Flavonoids inhibit COX-1 and COX-2 enzymes and cytokine/chemokine production in human whole blood," Inflammation, 2014.

[53] J.-D. Lee, J.-E. Huh, G. Jeon et al., "Flavonol-rich RVHxR from Rhus verniciflua Stokes and its major compound fisetin inhibits inflammation-related cytokines and angiogenic factor in rheumatoid arthritic fibroblast-like synovial cells and in vivo models," International Immunopharmacology, vol. 9, no. 3, pp. 268-276, 2009.

[54] G. O. dos Reis, G. Vicente, F. K. de Carvalho et al., "Croton antisyphiliticus Mart. attenuates the inflammatory response to carrageenan-induced pleurisy in mice," Inflammopharmacology, vol. 22, no. 2, pp. 115-126, 2014.

[55] R. Velagapudi, M. Aderogba, and O. A. Olajide, "Tiliroside, a dietary glycosidic flavonoid, inhibits TRAF-6/NF- $\kappa$ B/p38mediated neuroinflammation in activated BV2 microglia," Biochimica et Biophysica Acta, vol. 1840, no. 12, pp. 3311-3319, 2014.

[56] A. R. Kim, Q. Jin, H. G. Jin, H. J. Ko, and E. R. Woo, "Phenolic compounds with IL-6 inhibitory activity from Aster yomena," Archives of Pharmacal Research, vol. 37, no. 7, pp. 845-851, 2014.

[57] M. S. Kim and S. H. Kim, "Inhibitory effect of astragalin on expression of lipopolysaccharideinduced inflammatory mediators through NF- $\kappa \mathrm{B}$ in macrophages," Archives of Pharmacal Research, vol. 34, no. 12, pp. 2101-2107, 2011.

[58] Y.-Z. Yang, Y.-Z. Tang, and Y.-H. Liu, "Wogonoside displays anti-inflammatory effects through modulating inflammatory mediator expression using RAW264.7 cells," Journal of Ethnopharmacology, vol. 148, no. 1, pp. 271-276, 2013. 

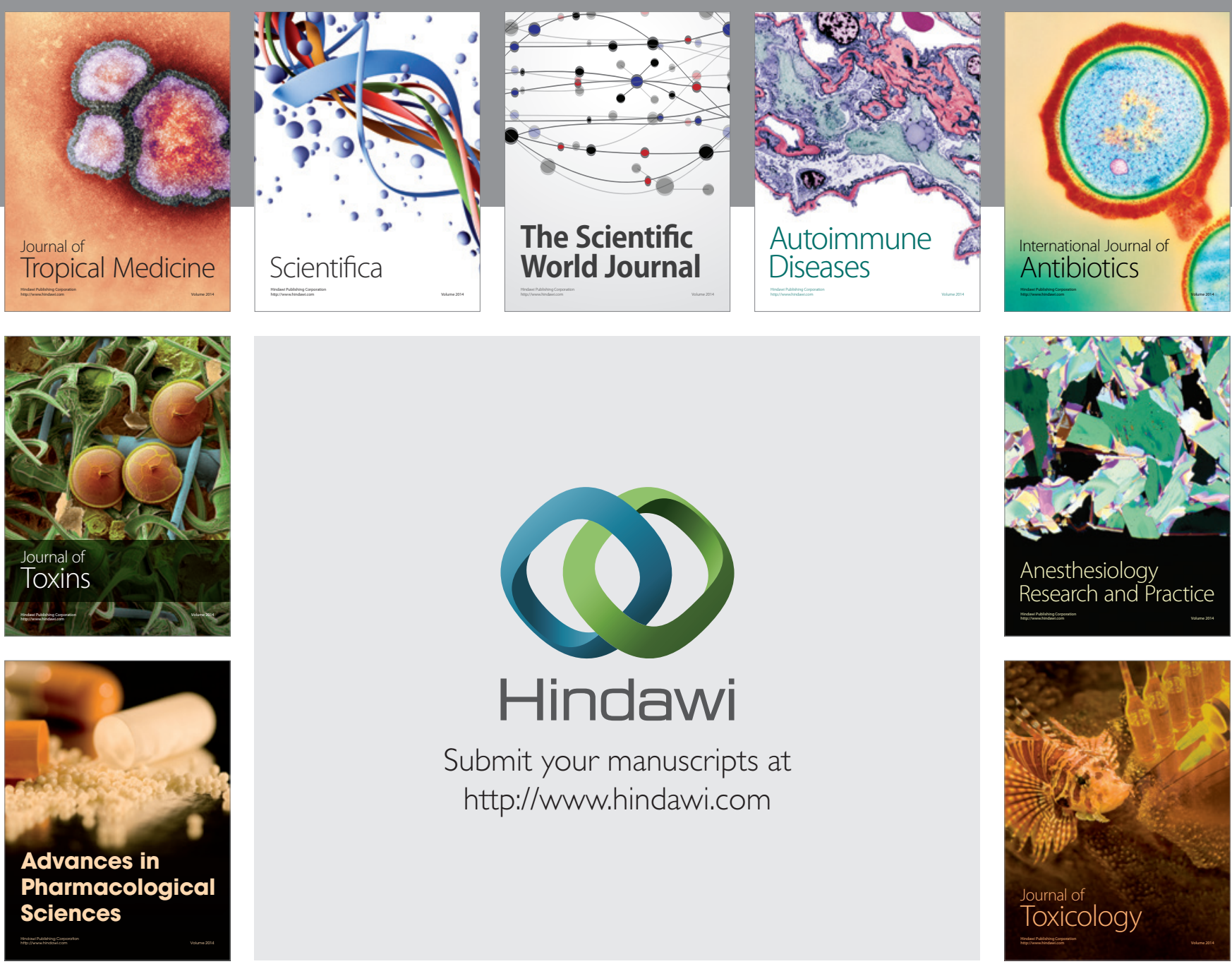

\section{Hindawi}

Submit your manuscripts at

http://www.hindawi.com
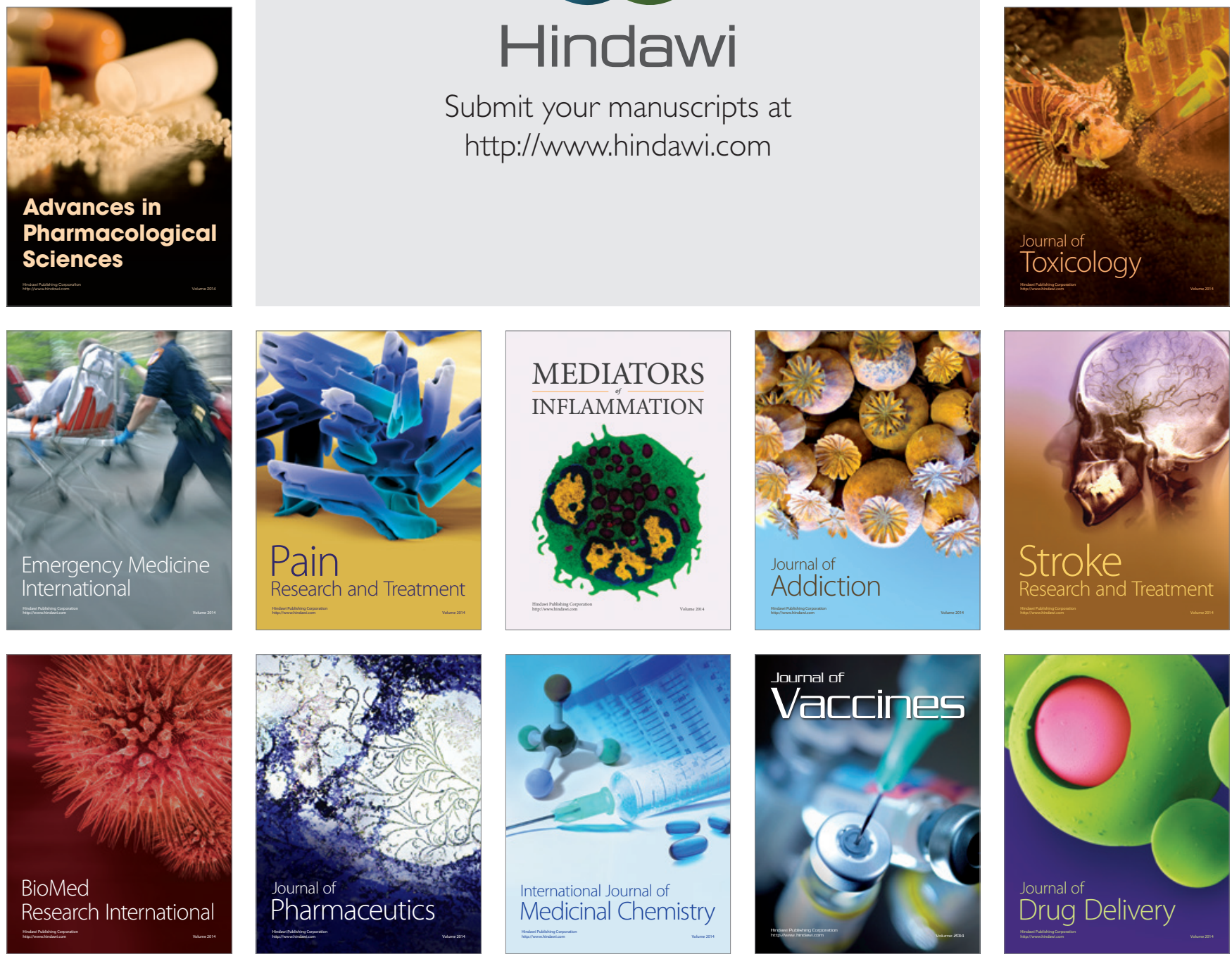